\title{
COMPARATIVE CHEMOMAPPING OF PHYTOCONSTITUENTS FROM DIFFERENT EXTRACTS OF GLOBE ARTICHOKE - CYNARA SCOLYMUS L.
}

\author{
SZABOLCS VÍGH ${ }^{a, b}$, ZOLTÁN CZIÁKY ${ }^{b}$, LÁSZLÓ TAMÁS SINKA ${ }^{b}$, \\ CIPRIAN PRIBAC ${ }^{c}$, LIANA MOŞ ${ }^{c}$, VIOLETA TURCUŞc, \\ JUDIT REMENYIK ${ }^{\mathrm{d}}$ and ENDRE MÁTHÉ ${ }^{\mathrm{b}, \mathrm{c}, \mathrm{d},{ }^{*}}$
}

\begin{abstract}
Artichoke (Cynara scolymus L.) is a well-known herb for its efficiency in the prevention/treatment of liver injuries, among other human chronic diseases. The aim of present study was to analyse the phytoconstituents content of aqueous and hydro-alcoholic extracts obtained from the leaves of artichoke. The chemomapping was carried out using UHPLC-ESI-MS. Several new and some known phytoconstituents were identified in the two type of extracts that have slightly different composition profiles. The newly found phytoconstituents in artichoke, plead for multiple health promoting effects that have presumably more stochastic than determinative features. Therefore, further experiments are needed using such extracts, and based on a system biology approach to clarify the complexity of beneficial effects of artichoke.
\end{abstract}

Keywords: globe artichoke, Cynara scolymus, phytoconstituents, bioactive compound, LC-ESI-MS

a University of Nyíregyháza, Institute of Agricultural Sciences, Sostói str. 31/B, H-4432, Nyíregyháza, Hungary (present address)

b University of Nyíregyháza, Agricultural and Molecular Research Institute, Sostói str. 31/B, H-4432, Nyíregyháza, Hungary

c "Vasile Goldiş" Western University of Arad, Faculty of Medicine, Liviu Rebreanu Str.91-93, RO-310414, Arad, Romania

d University of Debrecen, Faculty of Agriculture and Food Sciences and Environmental Management, Böszörményi str. 138, H-4032 Debrecen, Hungary

*Corresponding author: endre.mathe64@gmail.com 


\section{INTRODUCTION}

Globe artichoke (Cynara scolymus L.) has been cultivated since the ancient times in the Mediterranean and North African regions. In the middle ages, its cultivation spread across Western Europe from Italy to Spain, France, The Netherlands, England, and later on, in the 1800s reaches the Southern parts of USA. Moreover, Northern African countries like Egypt, Algeria and Tunisia together with South American countries like Argentina and Peru did become important artichoke producers in recent times.

Globe artichoke is considered a healthy food due to its nutritive and phytoconstituent content. It contains proteins, minerals, a low amount of lipids, dietary fibre and a high proportion of phenolics [1-2]. Among phenolics there were identified compounds like cynarin (1,3-di-O-caffeoylquinic acid), luteolin, cynaroside (luteolin-7-O-glucoside), scolymoside (luteolin-7-rutinoside); phenolic acids such as caffeic, coumaric, hydroxycinnamic, ferulic, caffeoylquinic acid derivatives; mono- and dicaffeoylquinic acids, including chlorogenic; acid alcohols; flavonoid glycosides [2-3]. The content of phytoconstituents was shown to vary among different cultivars and conditions related to cultivation, harvest, post-harvest and cooking [4-5].

Globe artichoke features a relatively high antioxidant capacity [6-7], its hepatoprotective, bile-enhancing and lipid-lowering effects have been demonstrated [8], while its implications in preventing cardiovascular disease by its lipidic and glycemic-reducing action has also been confirmed [9-10]. Moreover, its putative anticancer effect has been studied, and some experimental data suggests that artichoke extracts could be applied as a nonconventional, adjuvant therapy for cancer chemoprevention and/or treatment [11-13].

In the present paper we are describing the comparative UHPLCESI-MS chemomapping of aqueous and hydro-alcoholic artichoke extracts that were found to inhibit significantly the proliferation of several human cancer cell lines [14]. Our study was meant to identify all possible phytoconstituents with the used experimental setup, and as a consequence 49 and 51 molecules were described in the aqueous and hydro-alcoholic artichoke extracts, respectively. Some of the newly identified compounds were confirmed by standards, while other compounds have already been reported by others [15-26].

\section{RESULTS AND DISCUSSION}

In this paper, we are describing the qualitative analysis performed for artichoke (Cynara scolymus L.) extracts by applying reversed phase UHPLCESI-MS using a gradient mobile phase consisting of acetonitrile and water. The 
aqueous and the hydro-alcoholic extracts of artichoke leaves were investigated in positive and negative ionisation modes as described in Materials and Methods.

There have been 49 phytoconstituents identified in the aqueous artichoke extract as shown on the corresponding chromatograms (Figure 1-2.) and in Table 1.

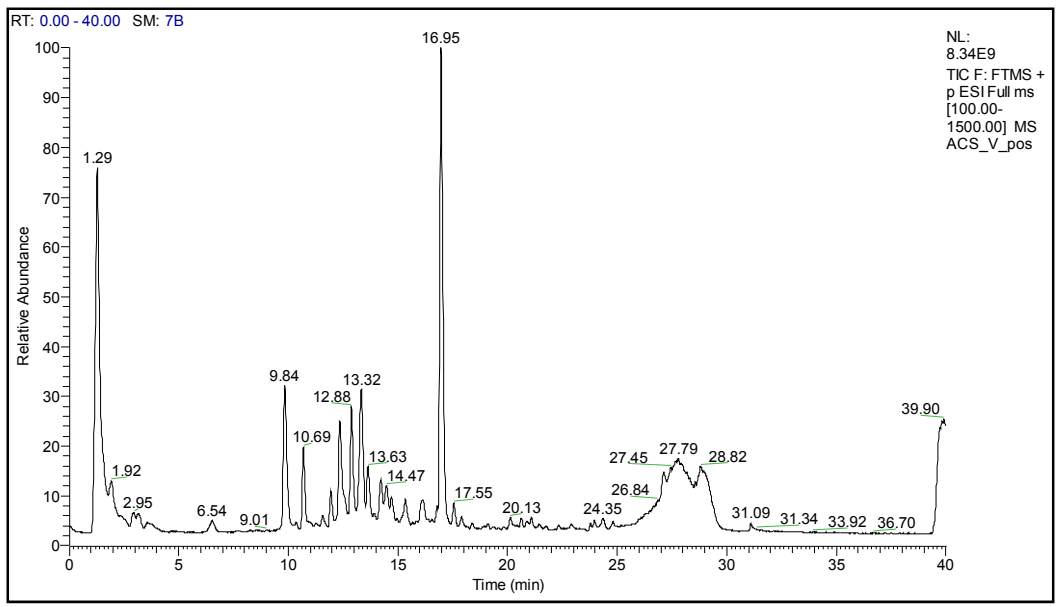

Figure 1. Total ion chromatogram of aqueous extract of artichoke in positive ionisation mode.

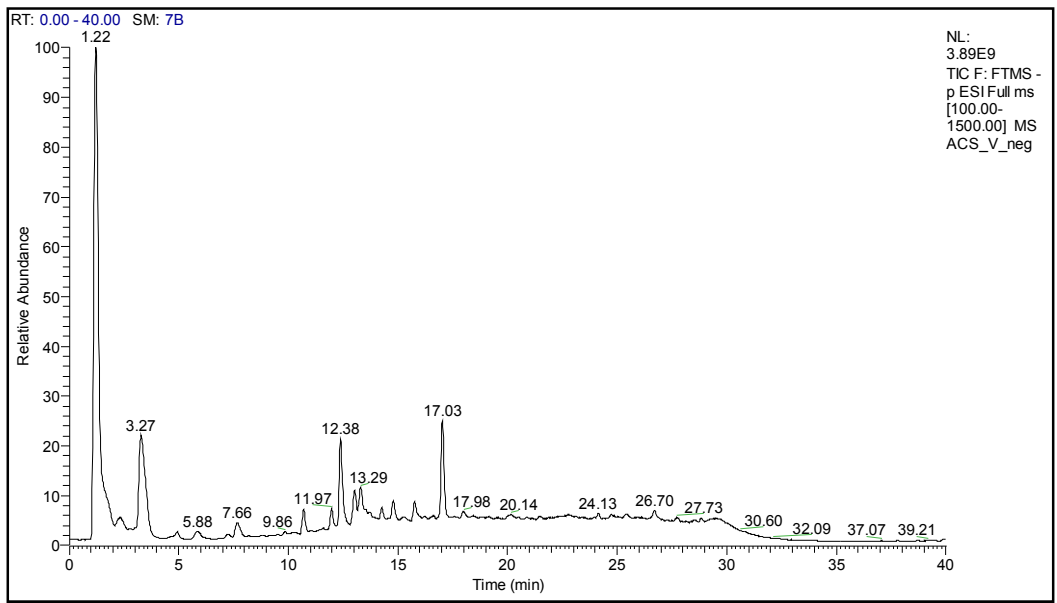

Figure 2. Total ion chromatogram of aqueous extract of artichoke in negative ionisation mode. 
Table 1. Phytoconstituents identified in the aqueous artichoke extract. $\mathrm{Rt}$-retention time; $[\mathrm{M}+\mathrm{H}]+-$ molecular ion masses; $[\mathrm{M}+\mathrm{H}]-$ - the found fragment ion mass; Ref- references; $\left.\left(^{*}\right)[\mathrm{M}]+;{ }^{* *}\right)$ confirmed by standards. The difference between measured and calculated molecular ion masses were always below 5 ppm.

\begin{tabular}{|c|c|c|c|c|c|c|c|}
\hline Peak & $\mathbf{R}_{\mathbf{t}}$ & {$[\mathrm{M}+\mathrm{H}]^{+}$} & {$[\mathrm{M}-\mathrm{H}]^{-}$} & Formula & $\begin{array}{c}\text { Fragments } \\
\text { found }\end{array}$ & Assignment & Ref. \\
\hline 1 & 1.22 & $104.10754^{*}$ & & $\mathrm{C}_{5} \mathrm{H}_{14} \mathrm{NO}$ & $\begin{array}{l}60.0814 \\
59.0736\end{array}$ & Choline & \\
\hline 2 & 1.27 & 175.11951 & & $\mathrm{C}_{6} \mathrm{H}_{14} \mathrm{~N}_{4} \mathrm{O}_{2}$ & $\begin{array}{l}158.0922, \\
1300975\end{array}$ & Arginine $^{\star \star}$ & \\
\hline 3 & 1.27 & & 179.05557 & $\mathrm{C}_{6} \mathrm{H}_{12} \mathrm{O}_{6}$ & $\begin{array}{l}113.0229, \\
101.0229\end{array}$ & $\begin{array}{l}\text { Glucose or } \\
\text { galactose }\end{array}$ & \\
\hline 4 & 1.29 & $138.05550^{*}$ & & $\mathrm{C}_{7} \mathrm{H}_{8} \mathrm{NO}_{2}$ & $\begin{array}{l}110.0602, \\
96.0447\end{array}$ & Trigonelline & \\
\hline 5 & 1.32 & 133.06132 & & $\mathrm{C}_{4} \mathrm{H}_{8} \mathrm{~N}_{2} \mathrm{O}_{3}$ & $\begin{array}{l}116.0344, \\
88.0397\end{array}$ & Asparagine $^{* *}$ & \\
\hline 6 & 1.43 & 324.05968 & & $\mathrm{C}_{9} \mathrm{H}_{13} \mathrm{~N}_{3} \mathrm{O}_{5}$ & $\begin{array}{l}112.0507, \\
95.0240\end{array}$ & Cytidine $^{* *}$ & \\
\hline 7 & 1.48 & 146.09296 & & $\mathrm{C}_{5} \mathrm{H}_{11} \mathrm{~N}_{3} \mathrm{O}_{2}$ & $\begin{array}{l}128.0817 \\
111.0555\end{array}$ & $\begin{array}{l}\text { 4- } \\
\text { Guanidinobutyric } \\
\text { acid }\end{array}$ & \\
\hline 8 & 1.51 & 136.06233 & & $\mathrm{C}_{5} \mathrm{H}_{5} \mathrm{~N}_{5}$ & $\begin{array}{l}119.0352, \\
94.0402\end{array}$ & \begin{tabular}{|l|} 
Adenine \\
\end{tabular} & \\
\hline 9 & 1.52 & & 362.05018 & $\mathrm{C}_{10} \mathrm{H}_{14} \mathrm{~N}_{5} \mathrm{O}_{8} \mathrm{P}$ & $\begin{array}{l}211.0005 \\
150.0408\end{array}$ & $\begin{array}{l}\text { Guanosine 5'- } \\
\text { monophosphate }\end{array}$ & \\
\hline 10 & 1.53 & 168.06607 & & $\mathrm{C}_{8} \mathrm{H}_{9} \mathrm{NO}_{3}$ & $\begin{array}{l}150.0548, \\
140.0705\end{array}$ & Pyridoxal $^{* *}$ & \\
\hline 11 & 1.57 & 124.03986 & & $\mathrm{C}_{6} \mathrm{H}_{5} \mathrm{NO}_{2}$ & $\begin{array}{l}96.0448 \\
80.0499\end{array}$ & 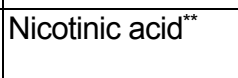 & \\
\hline 12 & 1.59 & $144.10245^{*}$ & & $\mathrm{C}_{7} \mathrm{H}_{14} \mathrm{NO}_{2}$ & $\begin{array}{l}\text { 102.0554, } \\
98.0968\end{array}$ & Stachydrine & \\
\hline 13 & 1.71 & 170.08172 & & $\mathrm{C}_{8} \mathrm{H}_{11} \mathrm{NO}_{3}$ & $\begin{array}{l}152.0704, \\
134.0600\end{array}$ & Pyridoxine $^{* *}$ & \\
\hline 14 & 1.74 & & 243.06171 & $\mathrm{C}_{9} \mathrm{H}_{12} \mathrm{~N}_{2} \mathrm{O}_{6}$ & $\begin{array}{l}200.0557, \\
153.0291\end{array}$ & Uridine & \\
\hline 15 & 1.76 & 113.03511 & & $\mathrm{C}_{4} \mathrm{H}_{4} \mathrm{~N}_{2} \mathrm{O}_{2}$ & $\begin{array}{l}96.0084 \\
95.0245\end{array}$ & Uracil $^{* *}$ & \\
\hline 16 & 1.78 & 182.08172 & & $\mathrm{C}_{9} \mathrm{H}_{11} \mathrm{NO}_{3}$ & \begin{tabular}{|l}
165.0544, \\
147.0439
\end{tabular} & \begin{tabular}{|l}
$2-$ \\
Hydroxyphenyl- \\
alanine
\end{tabular} & \\
\hline 17 & 1.92 & 123.05584 & & $\mathrm{C}_{6} \mathrm{H}_{6} \mathrm{~N}_{2} \mathrm{O}$ & $\begin{array}{l}106.0291 \\
96.0447\end{array}$ & Nicotinamide $^{* *}$ & \\
\hline 18 & 2.34 & & 346.05526 & $\mathrm{C}_{10} \mathrm{H}_{14} \mathrm{~N}_{5} \mathrm{O}_{7} \mathrm{P}$ & $\begin{array}{l}211.0006, \\
192.9902\end{array}$ & $\begin{array}{l}\text { Adenosine 5'- } \\
\text { monophosphate }\end{array}$ & \\
\hline 19 & 2.62 & & 282.08385 & $\mathrm{C}_{10} \mathrm{H}_{13} \mathrm{~N}_{5} \mathrm{O}_{5}$ & $\begin{array}{l}150.0408, \\
133.0143\end{array}$ & Guanosine & \\
\hline 20 & 2.94 & 268.10458 & & $\mathrm{C}_{10} \mathrm{H}_{13} \mathrm{~N}_{5} \mathrm{O}_{4}$ & $\begin{array}{l}136.0617, \\
119.0350\end{array}$ & Adenosine ${ }^{* *}$ & \\
\hline
\end{tabular}




\begin{tabular}{|c|c|c|c|c|c|c|c|}
\hline Peak & $\mathbf{R}_{\mathbf{t}}$ & {$[\mathrm{M}+\mathrm{H}]^{+}$} & {$[\mathrm{M}-\mathrm{H}]^{-}$} & Formula & $\begin{array}{c}\text { Fragments } \\
\text { found }\end{array}$ & Assignment & Ref. \\
\hline 21 & 3.18 & 166.08681 & & $\mathrm{C}_{9} \mathrm{H}_{11} \mathrm{NO}_{2}$ & $\begin{array}{l}149.0602 \\
131.0492\end{array}$ & Phenylalanine ${ }^{\star *}$ & \\
\hline 22 & 3.27 & & 353.08726 & $\mathrm{C}_{16} \mathrm{H}_{18} \mathrm{O}_{9}$ & $\begin{array}{l}191.0552, \\
179.0342 \\
\end{array}$ & $\begin{array}{l}\text { Caffeoylquinic } \\
\text { acid I }\end{array}$ & 16 \\
\hline 23 & 4,78 & 122.09698 & & $\mathrm{C}_{8} \mathrm{H}_{11} \mathrm{NO}_{2}$ & $\begin{array}{l}105.0702 \\
103.0546\end{array}$ & Phenethylamine & \\
\hline 24 & 4.83 & 220.11850 & & $\mathrm{C}_{9} \mathrm{H}_{17} \mathrm{NO}_{5}$ & $\begin{array}{l}202.1073 \\
184.0967\end{array}$ & $\begin{array}{l}\text { Pantothenic } \\
\text { acid }^{* *}\end{array}$ & \\
\hline 25 & 5.90 & & 337.09234 & $\mathrm{C}_{16} \mathrm{H}_{18} \mathrm{O}_{8}$ & $\begin{array}{l}191.0552 \\
163.0388\end{array}$ & $\begin{array}{l}\text { Coumaroylquinic } \\
\text { acid I }\end{array}$ & 17 \\
\hline 26 & 6.52 & 205.09771 & & $\mathrm{C}_{11} \mathrm{H}_{12} \mathrm{~N}_{2} \mathrm{O}_{2}$ & $\begin{array}{l}188.0705 \\
170.0599\end{array}$ & Tryptophan $^{* *}$ & \\
\hline 27 & 7.66 & & 353.08726 & $\mathrm{C}_{16} \mathrm{H}_{18} \mathrm{O}_{9}$ & \begin{tabular}{|l}
191.0552 \\
179.0338
\end{tabular} & $\begin{array}{l}\text { Caffeoylquinic } \\
\text { acid II }\end{array}$ & 16 \\
\hline 28 & 8.31 & 190.05042 & & $\mathrm{C}_{10} \mathrm{H}_{7} \mathrm{NO}_{3}$ & $\begin{array}{l}162.0547 \\
144.0442\end{array}$ & Kynurenic acid & \\
\hline 29 & 8.84 & 341.08726 & & $\mathrm{C}_{15} \mathrm{H}_{16} \mathrm{O}_{9}$ & $\begin{array}{l}179.0338, \\
151.0388\end{array}$ & Esculin & \\
\hline 30 & 8.86 & & 335.07669 & $\mathrm{C}_{16} \mathrm{H}_{16} \mathrm{O}_{8}$ & \begin{tabular}{|l}
179.0339 \\
161.0231 \\
\end{tabular} & $\begin{array}{l}\text { Caffeoylshikimic } \\
\text { acid I }\end{array}$ & \\
\hline 31 & 9.57 & 295.12940 & & $\mathrm{C}_{14} \mathrm{H}_{18} \mathrm{~N}_{2} \mathrm{O}_{5}$ & $\begin{array}{l}278.1119, \\
232.0961\end{array}$ & $\begin{array}{l}Y^{-} \\
\text {Glutamylphenyl- } \\
\text { alanine }\end{array}$ & \\
\hline 32 & 9.97 & 298.09739 & & $\mathrm{C}_{11} \mathrm{H}_{15} \mathrm{~N}_{5} \mathrm{O}_{3} \mathrm{~S}$ & $\begin{array}{l}163.0422 \\
145.0313\end{array}$ & $\begin{array}{l}\text { 5'-S-Methyl-5'- } \\
\text { thioadenosine }\end{array}$ & \\
\hline 33 & 10.19 & & 337.09234 & $\mathrm{C}_{16} \mathrm{H}_{18} \mathrm{O}_{8}$ & $\begin{array}{l}191.0552, \\
163.0389\end{array}$ & $\begin{array}{l}\text { Coumaroylquinic } \\
\text { acid II }\end{array}$ & 17 \\
\hline 34 & 11.53 & 191.07082 & & $\mathrm{C}_{11} \mathrm{H}_{10} \mathrm{O}_{3}$ & $\begin{array}{l}176.0466 \\
148.0518\end{array}$ & $\begin{array}{l}\text { 7-Methoxy-4- } \\
\text { methylcoumarin }\end{array}$ & \\
\hline 35 & 12.78 & & 593.15065 & $\mathrm{C}_{21} \mathrm{H}_{18} \mathrm{O}_{11}$ & $\begin{array}{l}473.1093 \\
383.0772\end{array}$ & Vicenin-2 & \\
\hline 36 & 13.02 & & 335.07669 & $\mathrm{C}_{16} \mathrm{H}_{16} \mathrm{O}_{8}$ & \begin{tabular}{|l}
179.0339, \\
161.0232 \\
\end{tabular} & $\begin{array}{l}\text { Caffeoylshikimic } \\
\text { acid III }\end{array}$ & \\
\hline 37 & 13.02 & & 515.11896 & $\mathrm{C}_{25} \mathrm{H}_{24} \mathrm{O}_{12}$ & $\begin{array}{l}335.0776 \\
191.0552\end{array}$ & $\begin{array}{l}\text { 1,3-Di-O- } \\
\text { caffeoylquinic } \\
\text { acid (Cynarin) }\end{array}$ & \\
\hline 38 & 13.27 & 283.15455 & & $\mathrm{C}_{15} \mathrm{H}_{22} \mathrm{O}_{5}$ & $\begin{array}{l}265.1429, \\
247.1324\end{array}$ & Cynaratriol & \\
\hline 39 & 13.54 & & 461.07201 & $\mathrm{C}_{21} \mathrm{H}_{18} \mathrm{O}_{12}$ & $\begin{array}{l}285.0404, \\
217.0501\end{array}$ & $\begin{array}{l}\text { Luteolin-7-O- } \\
\text { glucuronide }\end{array}$ & 18 \\
\hline 40 & 13.54 & 146.06059 & & $\mathrm{C}_{9} \mathrm{H}_{7} \mathrm{NO}$ & \begin{tabular}{|l|}
$118.0652,1$ \\
17.0573 \\
\end{tabular} & \begin{tabular}{|l|} 
Indole-4- \\
carbaldehyde
\end{tabular} & \\
\hline 41 & 13.81 & 179.07082 & & $\mathrm{C}_{10} \mathrm{H}_{10} \mathrm{O}_{3}$ & \begin{tabular}{|l|}
161.0594, \\
147.0438
\end{tabular} & $\begin{array}{l}\text { 4-Hydroxy-3- } \\
\text { methoxy- } \\
\text { cinnamaldehyde }\end{array}$ & \\
\hline 42 & 14.60 & & 445.07709 & $\mathrm{C}_{21} \mathrm{H}_{18} \mathrm{O}_{11}$ & $\begin{array}{l}269.0454, \\
225.0546\end{array}$ & \begin{tabular}{|l|} 
Apigenin-7-O- \\
glucuronide
\end{tabular} & 19 \\
\hline
\end{tabular}


SZ. VÍGH, Z. CZIÁKY, L. T. SINKA, C. PRIBAC, L. MOŞ, V. TURCUŞ, J. REMENYIK, E. MÁTHÉ

\begin{tabular}{|c|c|c|c|c|c|c|c|}
\hline Peak & $\mathbf{R}_{\mathbf{t}}$ & {$[\mathrm{M}+\mathrm{H}]^{+}$} & {$[\mathrm{M}-\mathrm{H}]^{-}$} & Formula & $\begin{array}{c}\text { Fragments } \\
\text { found }\end{array}$ & Assignment & Ref. \\
\hline 43 & 14.74 & & 593.15065 & $\mathrm{C}_{27} \mathrm{H}_{30} \mathrm{O}_{15}$ & $\begin{array}{l}285.0404 \\
133.0275\end{array}$ & $\begin{array}{l}\text { Luteolin-7-O- } \\
\text { rutinoside } \\
\text { (Scolymoside) }\end{array}$ & 20 \\
\hline 44 & 14.79 & & 447.09274 & $\mathrm{C}_{21} \mathrm{H}_{20} \mathrm{O}_{11}$ & $\begin{array}{l}327.0509 \\
285.0403\end{array}$ & $\begin{array}{l}\text { Luteolin-7-O- } \\
\text { glucoside } \\
\text { (Cynaroside) }\end{array}$ & $\begin{array}{l}18, \\
19,21\end{array}$ \\
\hline 45 & 15.20 & & 193.05009 & $\mathrm{C}_{10} \mathrm{H}_{10} \mathrm{O}_{4}$ & $\begin{array}{l}178.0262 \\
149.0596\end{array}$ & Ferulic acid & \\
\hline 46 & 15.67 & 433.11347 & & $\mathrm{C}_{21} \mathrm{H}_{20} \mathrm{O}_{10}$ & $\begin{array}{l}\text { 271.0600, } \\
153.0180\end{array}$ & $\begin{array}{l}\text { Cosmosiin } \\
\text { (Apigenin-7-O- } \\
\text { glucoside) }\end{array}$ & 22,23 \\
\hline 47 & 17.98 & & 285.03991 & $\mathrm{C}_{15} \mathrm{H}_{10} \mathrm{O}_{6}$ & \begin{tabular}{|l}
217.0499 \\
199.0393
\end{tabular} & Luteolin & \\
\hline 48 & 20.34 & & 809.43235 & $\mathrm{C}_{42} \mathrm{H}_{66} \mathrm{O}_{15}$ & $\begin{array}{l}\text { 647.3814, } \\
603.3902 \\
\end{array}$ & $\begin{array}{l}\text { Cynarasaponin } \\
\text { E }\end{array}$ & 26 \\
\hline 49 & 21.86 & & 793.43744 & $\mathrm{C}_{42} \mathrm{H}_{66} \mathrm{O}_{14}$ & $\begin{array}{l}631.3859 \\
587.3961\end{array}$ & $\begin{array}{l}\text { Cynarasaponin } \\
\text { C }\end{array}$ & 26 \\
\hline
\end{tabular}

There have been 51 phytoconstituents identified in the hydro-alcoholic artichoke extract as shown on Fig.3-4 and in Table 2.

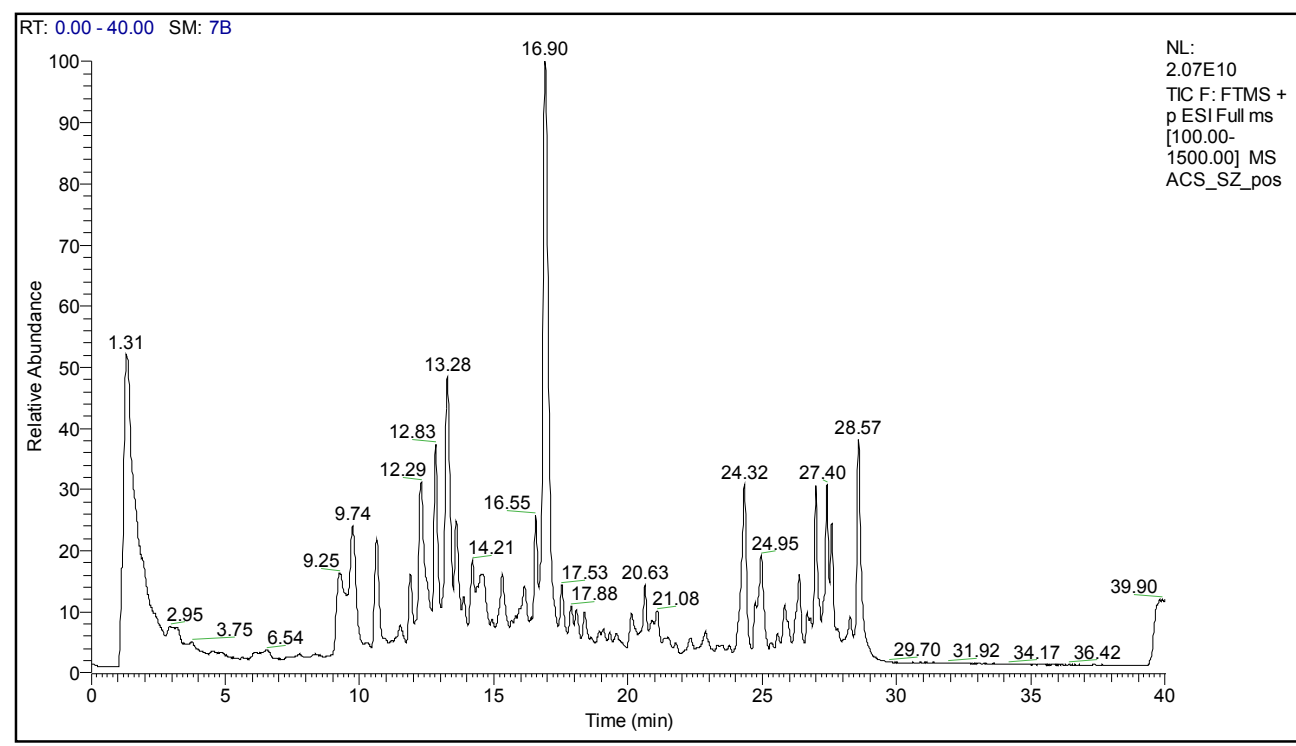

Figure 3. Total ion chromatogram of hydro-alcoholic extract of artichoke in positive ionisation mode. 


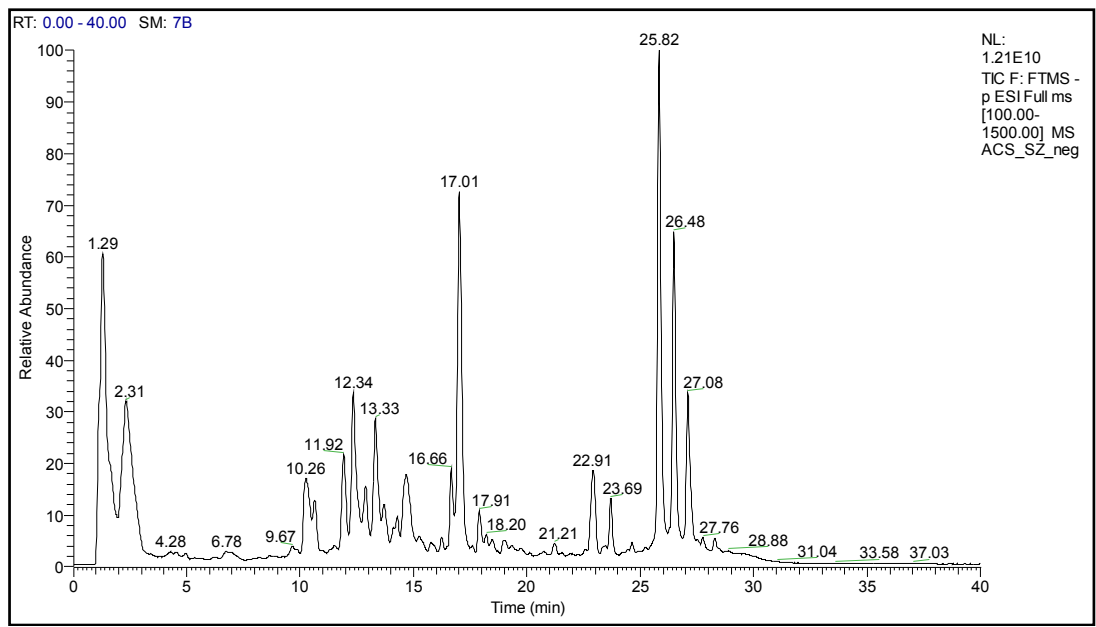

Figure 4. Total ion chromatogram of hydro-alcoholic extract of artichoke in negative ionisation mode.

Table 2. Phytoconstituents identified in the hydro-alcoholic artichoke extract.

$\mathrm{Rt}$-retention time; $[\mathrm{M}+\mathrm{H}]+-$ molecular ion masses; $[\mathrm{M}+\mathrm{H}]-$ - the found fragment ion mass; Ref- references; $\left(^{*}\right)[\mathrm{M}]+;\left(^{* *}\right)$ confirmed by standards. The difference between measured and calculated molecular ion masses were always below 5 ppm.

\begin{tabular}{|c|c|c|c|c|c|c|c|}
\hline Peak & $\mathbf{R}_{\mathrm{t}}$ & {$[\mathrm{M}+\mathrm{H}]^{+}$} & {$[\mathrm{M}-\mathrm{H}]^{-}$} & Formula & $\begin{array}{c}\text { Fragments } \\
\text { found }\end{array}$ & Assignment & Ref. \\
\hline 1 & 1.26 & $138.05550^{*}$ & & $\mathrm{C}_{7} \mathrm{H}_{8} \mathrm{NO}_{2}$ & $\begin{array}{l}110.0603 \\
96.0449\end{array}$ & Trigonelline & \\
\hline 2 & 1.28 & $104.10754^{*}$ & & $\mathrm{C}_{5} \mathrm{H}_{14} \mathrm{NO}$ & $\begin{array}{l}60.0814 \\
59.0736\end{array}$ & Choline & \\
\hline 3 & 1.30 & 175.11951 & & $\mathrm{C}_{6} \mathrm{H}_{14} \mathrm{~N}_{4} \mathrm{O}_{2}$ & $\begin{array}{l}158.0923 \\
130.0976\end{array}$ & Arginine ${ }^{* *}$ & \\
\hline 4 & 1.35 & 133.06132 & & $\mathrm{C}_{4} \mathrm{H}_{8} \mathrm{~N}_{2} \mathrm{O}_{3}$ & $\begin{array}{l}116.0343 \\
88.0397\end{array}$ & Asparagine $^{* *}$ & \\
\hline 5 & 1.38 & & 179.05557 & $\mathrm{C}_{6} \mathrm{H}_{12} \mathrm{O}_{6}$ & $\begin{array}{l}113.0229 \\
101.0230\end{array}$ & $\begin{array}{l}\text { Glucose or } \\
\text { galactose }\end{array}$ & \\
\hline 6 & 1.50 & 324.05968 & & $\mathrm{C}_{9} \mathrm{H}_{13} \mathrm{~N}_{3} \mathrm{O}_{5}$ & $\begin{array}{l}112.0507 \\
95.0243\end{array}$ & Cytidine $^{* *}$ & \\
\hline 7 & 1.52 & 146.09296 & & $\mathrm{C}_{5} \mathrm{H}_{11} \mathrm{~N}_{3} \mathrm{O}_{2}$ & $\begin{array}{l}128.0815 \\
111.0554\end{array}$ & $\begin{array}{l}\text { 4- } \\
\text { Guanidinobutyric } \\
\text { acid }\end{array}$ & \\
\hline 8 & 1.53 & 136.06233 & & $\mathrm{C}_{5} \mathrm{H}_{5} \mathrm{~N}_{5}$ & $\begin{array}{l}119.0353, \\
94.0403\end{array}$ & Adenine & \\
\hline 9 & 1.62 & 124.03986 & & $\mathrm{C}_{6} \mathrm{H}_{5} \mathrm{NO}_{2}$ & $\begin{array}{l}96.0448 \\
80.0500\end{array}$ & ${\text { Nicotinic } \text { acid }^{* *}}^{*}$ & \\
\hline
\end{tabular}


SZ. VÍGH, Z. CZIÁKY, L. T. SINKA, C. PRIBAC, L. MOŞ, V. TURCUŞ, J. REMENYIK, E. MÁTHÉ

\begin{tabular}{|c|c|c|c|c|c|c|c|}
\hline Peak & $\mathbf{R}_{\mathbf{t}}$ & {$[\mathrm{M}+\mathrm{H}]^{+}$} & {$[\mathrm{M}-\mathrm{H}]^{-}$} & Formula & $\begin{array}{c}\text { Fragments } \\
\text { found }\end{array}$ & Assignment & Ref. \\
\hline 10 & 1.75 & 170.08172 & & $\mathrm{C}_{8} \mathrm{H}_{11} \mathrm{NO}_{3}$ & $\begin{array}{l}152.0704, \\
134.0601\end{array}$ & Pyridoxine $^{* *}$ & \\
\hline 11 & 1.83 & 113.03511 & & $\mathrm{C}_{4} \mathrm{H}_{4} \mathrm{~N}_{2} \mathrm{O}_{2}$ & $\begin{array}{l}96.0084 \\
95.0245\end{array}$ & Uracil $^{* *}$ & \\
\hline 12 & 1.84 & 182.08172 & & $\mathrm{C}_{9} \mathrm{H}_{11} \mathrm{NO}_{3}$ & $\begin{array}{l}165.0542, \\
147.0439\end{array}$ & \begin{tabular}{|l|}
$2-$ \\
Hydroxyphenyl- \\
alanine
\end{tabular} & \\
\hline 13 & 1.94 & 123.05584 & & $\mathrm{C}_{6} \mathrm{H}_{6} \mathrm{~N}_{2} \mathrm{O}$ & $\begin{array}{l}\text { 106.0290, } \\
96.0448\end{array}$ & Nicotinamide $^{* *}$ & \\
\hline 14 & 2.68 & & 282.08385 & $\mathrm{C}_{10} \mathrm{H}_{13} \mathrm{~N}_{5} \mathrm{O}_{5}$ & $\begin{array}{l}150.0408, \\
133.0142\end{array}$ & Guanosine & \\
\hline 15 & 2.99 & 268.10458 & & $\mathrm{C}_{10} \mathrm{H}_{13} \mathrm{~N}_{5} \mathrm{O}_{4}$ & $\begin{array}{l}136.0617 \\
119.0347\end{array}$ & Adenosine ${ }^{* \star}$ & \\
\hline 16 & 3.23 & 166.08681 & & $\mathrm{C}_{9} \mathrm{H}_{11} \mathrm{NO}_{2}$ & $\begin{array}{l}149.0601, \\
131.0493\end{array}$ & Phenylalanine ${ }^{* *}$ & \\
\hline 17 & 4.85 & 122.09698 & & $\mathrm{C}_{8} \mathrm{H}_{11} \mathrm{~N}$ & $\begin{array}{l}105.0702, \\
103.0546\end{array}$ & Phenethylamine & \\
\hline 18 & 4.87 & 220.11850 & & $\mathrm{C}_{9} \mathrm{H}_{17} \mathrm{NO}_{5}$ & $\begin{array}{l}\text { 202.1070, } \\
184.0967\end{array}$ & $\begin{array}{l}\text { Pantothenic } \\
\text { acid }^{* *}\end{array}$ & \\
\hline 19 & 6.54 & 205.09771 & & $\mathrm{C}_{11} \mathrm{H}_{12} \mathrm{~N}_{2} \mathrm{O}_{2}$ & $\begin{array}{l}188.0705, \\
170.0598\end{array}$ & Tryptophan $^{* *}$ & \\
\hline 20 & 8.26 & 190.05042 & & $\mathrm{C}_{10} \mathrm{H}_{7} \mathrm{NO}_{3}$ & $\begin{array}{l}162.0547, \\
144.0442\end{array}$ & Kynurenic acid & \\
\hline 21 & 8.79 & 341.08726 & & $\mathrm{C}_{15} \mathrm{H}_{16} \mathrm{O}_{9}$ & $\begin{array}{l}179.0337 \\
151.0389\end{array}$ & Esculin & \\
\hline 22 & 9.55 & 295.12940 & & $\mathrm{C}_{14} \mathrm{H}_{18} \mathrm{~N}_{2} \mathrm{O}_{5}$ & $\begin{array}{l}278.1121 \\
232.0964\end{array}$ & $\begin{array}{l}- \\
\text { Glutamylphenyl- } \\
\text { alanine }\end{array}$ & \\
\hline 23 & 9.95 & 298.09739 & & $\mathrm{C}_{11} \mathrm{H}_{15} \mathrm{~N}_{5} \mathrm{O}_{3} \mathrm{~S}$ & $\begin{array}{l}163.0422, \\
145.0318\end{array}$ & $\begin{array}{l}\text { 5'-S-Methyl-5'- } \\
\text { thioadenosine }\end{array}$ & \\
\hline 24 & 11.49 & 174.11302 & & $\mathrm{C}_{8} \mathrm{H}_{15} \mathrm{NO}_{3}$ & $\begin{array}{l}156.1010 \\
132.1019\end{array}$ & $\begin{array}{l}\mathrm{N}- \\
\text { Acetylisoleucine }\end{array}$ & \\
\hline 25 & 11.51 & 191.07082 & & $\mathrm{C}_{11} \mathrm{H}_{10} \mathrm{O}_{3}$ & $\begin{array}{l}176.0462 \\
148.0517\end{array}$ & $\begin{array}{l}\text { 7-Methoxy-4- } \\
\text { methylcoumarin }\end{array}$ & \\
\hline 26 & 12.03 & 174.11302 & & $\mathrm{C}_{8} \mathrm{H}_{15} \mathrm{NO}_{3}$ & $\begin{array}{l}156.1012 \\
132.1019\end{array}$ & N-Acetylleucine & \\
\hline 27 & 12.52 & & 593.15065 & $\mathrm{C}_{21} \mathrm{H}_{18} \mathrm{O}_{11}$ & $\begin{array}{l}473.1084, \\
383.0770\end{array}$ & Vicenin-2 & \\
\hline 28 & 12.57 & & 461.07201 & $\mathrm{C}_{21} \mathrm{H}_{18} \mathrm{O}_{12}$ & $\begin{array}{l}285.0403, \\
217.0499\end{array}$ & $\begin{array}{l}\text { Luteolin-7-O- } \\
\text { glucuronide }\end{array}$ & 18 \\
\hline 29 & 12.86 & 193.05009 & & $\mathrm{C}_{10} \mathrm{H}_{8} \mathrm{O}_{4}$ & $\begin{array}{l}\text { 178.0258, } \\
165.0544\end{array}$ & Scopoletin & \\
\hline 30 & 13.05 & & 515.11896 & $\mathrm{C}_{25} \mathrm{H}_{24} \mathrm{O}_{12}$ & $\begin{array}{l}335.0770, \\
191.0552\end{array}$ & $\begin{array}{l}\text { 1,3-Di-O- } \\
\text { caffeoylquinic } \\
\text { acid (Cynarin) }\end{array}$ & \\
\hline 31 & 13.27 & 283.15455 & & $\mathrm{C}_{15} \mathrm{H}_{22} \mathrm{O}_{5}$ & $\begin{array}{l}265.1429, \\
247.1324\end{array}$ & Cynaratriol & \\
\hline
\end{tabular}




\begin{tabular}{|c|c|c|c|c|c|c|c|}
\hline Peak & $\mathbf{R}_{\mathbf{t}}$ & {$[\mathrm{M}+\mathrm{H}]^{+}$} & {$[\mathrm{M}-\mathrm{H}]^{-}$} & Formula & $\begin{array}{c}\text { Fragments } \\
\text { found }\end{array}$ & Assignment & Ref. \\
\hline 32 & 13.32 & 163.07591 & & $\mathrm{C}_{10} \mathrm{H}_{10} \mathrm{O}_{2}$ & \begin{tabular}{|l|}
131.0492, \\
103.0545 \\
\end{tabular} & $\begin{array}{l}\text { Methyl } \\
\text { cinnamate } \\
\end{array}$ & \\
\hline 33 & 13.38 & & 581.18703 & $\mathrm{C}_{27} \mathrm{H}_{34} \mathrm{O}_{14}$ & \begin{tabular}{|l|}
297.1768, \\
167.0337 \\
\end{tabular} & $\begin{array}{l}\text { Naringin } \\
\text { dihydrochalcone }\end{array}$ & \\
\hline 34 & 13.52 & 146.06059 & & $\mathrm{C}_{9} \mathrm{H}_{7} \mathrm{NO}$ & $\begin{array}{l}118.0652,1 \\
17.0572\end{array}$ & \begin{tabular}{|l|} 
Indole-4- \\
carbaldehyde
\end{tabular} & \\
\hline 35 & 13.65 & & 445.07709 & $\mathrm{C}_{21} \mathrm{H}_{18} \mathrm{O}_{11}$ & $\begin{array}{l}269.0454, \\
225.0550\end{array}$ & $\begin{array}{l}\text { Apigenin-7-O- } \\
\text { glucuronide }\end{array}$ & 19 \\
\hline 36 & 13.80 & 179.07082 & & $\mathrm{C}_{10} \mathrm{H}_{10} \mathrm{O}_{3}$ & $\begin{array}{l}\text { 161.0595, } \\
147.0439\end{array}$ & $\begin{array}{l}\text { 4-Hydroxy-3- } \\
\text { methoxy- } \\
\text { cinnamaldehyde }\end{array}$ & \\
\hline 37 & 14.71 & & 593.15065 & $\mathrm{C}_{27} \mathrm{H}_{30} \mathrm{O}_{15}$ & $\begin{array}{l}285.0404 \\
133.0279\end{array}$ & $\begin{array}{l}\text { Luteolin-7-O- } \\
\text { rutinoside } \\
\text { (Scolymoside) }\end{array}$ & 20 \\
\hline 38 & 14.73 & & 447.09274 & $\mathrm{C}_{21} \mathrm{H}_{20} \mathrm{O}_{11}$ & $\begin{array}{l}327.0507 \\
285.0400\end{array}$ & $\begin{array}{l}\text { Luteolin-7-O- } \\
\text { glucoside } \\
\text { (Cynaroside) } \\
\end{array}$ & $\begin{array}{l}18, \\
19,21\end{array}$ \\
\hline 39 & 15.19 & & 193.05009 & $\mathrm{C}_{10} \mathrm{H}_{10} \mathrm{O}_{4}$ & $\begin{array}{l}178.0259, \\
149.0595\end{array}$ & Ferulic acid & \\
\hline 40 & 15.22 & & 269.04500 & $\mathrm{C}_{15} \mathrm{H}_{10} \mathrm{O}_{5}$ & $\begin{array}{l}225.0550, \\
201.0550\end{array}$ & Apigenin $^{* *}$ & 21,24 \\
\hline 41 & 15.54 & 579.17139 & & $\mathrm{C}_{27} \mathrm{H}_{30} \mathrm{O}_{14}$ & $\begin{array}{l}271.0595 \\
153.0176\end{array}$ & $\begin{array}{l}\text { Isorhoifolin } \\
\text { (Apigenin 7-O- } \\
\text { rutinoside) }\end{array}$ & 25 \\
\hline 42 & 15.66 & 433.11347 & & $\mathrm{C}_{21} \mathrm{H}_{20} \mathrm{O}_{10}$ & $\begin{array}{l}271.0594, \\
153.0179\end{array}$ & $\begin{array}{l}\text { Cosmosiin } \\
\text { (Apigenin-7-O- } \\
\text { glucoside) }\end{array}$ & 22,23 \\
\hline 43 & 17.89 & & 285.03991 & $\mathrm{C}_{15} \mathrm{H}_{10} \mathrm{O}_{6}$ & \begin{tabular}{|l}
217.0498, \\
199.0391 \\
\end{tabular} & Luteolin & \\
\hline 44 & 18.94 & & 539.04618 & $\mathrm{C}_{25} \mathrm{H}_{16} \mathrm{O}_{14}$ & $\begin{array}{l}269.0453 \\
201.0548\end{array}$ & $\begin{array}{l}\text { Unknown } \\
\text { Apigenin } \\
\text { derivative }\end{array}$ & \\
\hline 45 & 19.03 & 301.07121 & & $\mathrm{C}_{16} \mathrm{H}_{12} \mathrm{O}_{6}$ & $\begin{array}{l}286.0466, \\
258.0515\end{array}$ & Diosmetin & \\
\hline 46 & 21.76 & 329.10251 & & $\mathrm{C}_{18} \mathrm{H}_{16} \mathrm{O}_{6}$ & \begin{tabular}{|l|}
314.0781, \\
313.0697 \\
\end{tabular} & Salvigenin & \\
\hline 47 & 25.38 & 291.23241 & & $\mathrm{C}_{19} \mathrm{H}_{30} \mathrm{O}_{2}$ & $\begin{array}{l}\text { 259.2035, } \\
241.1949\end{array}$ & $\begin{array}{l}\text { Stearidonic acid } \\
\text { methyl ester }\end{array}$ & \\
\hline 48 & 25.85 & 305.24806 & & $\mathrm{C}_{20} \mathrm{H}_{32} \mathrm{O}_{2}$ & $\begin{array}{l}259.2058, \\
241.1939\end{array}$ & $\begin{array}{l}\text { Stearidonic acid } \\
\text { ethyl ester }\end{array}$ & \\
\hline 49 & 27.20 & 457.36818 & & $\mathrm{C}_{30} \mathrm{H}_{48} \mathrm{O}_{3}$ & $\begin{array}{l}439.3553 \\
411.3619\end{array}$ & Ursolic acid & \\
\hline 50 & 19.43 & & 809.43235 & $\mathrm{C}_{42} \mathrm{H}_{66} \mathrm{O}_{15}$ & $\begin{array}{l}647.3806, \\
603.3903\end{array}$ & $\begin{array}{l}\text { Cynarasaponin } \\
\text { E }\end{array}$ & 26 \\
\hline 51 & 21.20 & & 793.43744 & $\mathrm{C}_{42} \mathrm{H}_{66} \mathrm{O}_{14}$ & $\begin{array}{l}\text { 631.3851, } \\
587.3955\end{array}$ & $\begin{array}{l}\text { Cynarasaponin } \\
\text { C }\end{array}$ & 26 \\
\hline
\end{tabular}


The identification of the phytoconstituents was achieved by comparing individually the retention time, accurate mass, isotopic distribution and fragmentation pattern of every single newly detected molecule with artichoke compounds already reported in literature, and by screening MS databases like Metlin, mzCloud and Massbank. The identified molecules belong to twelve classes of phytoconstituents, and besides similarities, there are some striking differences among the aqueous and hydro-alcoholic artichoke extracts regarding their content as summarized in Table 3.

Table 3. Phytoconstituents identified in the aqueous and hydro-alcoholic artichoke extracts.

\begin{tabular}{|c|c|c|c|}
\hline \multicolumn{2}{|r|}{ Phytoconstituents } & \multirow{2}{*}{$\begin{array}{c}\begin{array}{c}\text { Aqueous } \\
\text { artichoke }\end{array} \\
+\end{array}$} & \multirow{2}{*}{\begin{tabular}{|c|}
$\begin{array}{c}\text { Hydro-alcoholic } \\
\text { artichoke }\end{array}$ \\
+
\end{tabular}} \\
\hline Alkaloids & Kynurenic acid & & \\
\hline & Trigonelline & + & + \\
\hline & Stachydrine $^{a}$ & + & \\
\hline \multirow[t]{9}{*}{ Aminoacids } & 2-Hydroxyphenylalanine & + & + \\
\hline & 4-Guanidinobutyric acid & + & + \\
\hline & Arginine & + & + \\
\hline & Asparagine & + & + \\
\hline & L-Phenylalanine & + & + \\
\hline & Y-Glutamilphenylalanin & + & + \\
\hline & Tryptophan & + & + \\
\hline & N-Acetylisoleucine ${ }^{b}$ & & + \\
\hline & N-Acetylleucin ${ }^{b}$ & & + \\
\hline \multirow[t]{3}{*}{ Coumarins } & 7-Methoxy-4-methylcoumarin & + & + \\
\hline & $\begin{array}{l}\text { 4-hidroxy-3-methoxy- } \\
\text { cinnamaldehyde }\end{array}$ & + & + \\
\hline & Scopoletin $^{\mathrm{b}}$ & & + \\
\hline \multirow[t]{13}{*}{ Flavonoids } & Unknown Apigenin derivative ${ }^{b}$ & & + \\
\hline & Apigenin $^{\mathrm{b}}$ & & + \\
\hline & Cosmosiin (Apigenin-7-O-glucoside) & + & + \\
\hline & Diosmetin $^{\mathrm{b}}$ & & + \\
\hline & Luteolin & + & + \\
\hline & Luteolin-7-O-glucoside (cynaroside) & + & + \\
\hline & Luteolin-7-O-glucuronide & + & + \\
\hline & Apigenin-7-O-glucuronide & + & + \\
\hline & Luteolin-7-O-rutinoside (scolymoside) & + & + \\
\hline & $\begin{array}{l}\text { lsorhoifolin (Apigenin-7-O- } \\
\text { rutinoside) }\end{array}$ & & + \\
\hline & Salvigenin ${ }^{\mathrm{b}}$ & & + \\
\hline & Naringin dihydrochalcone $\mathrm{e}^{\mathrm{b}}$ & & + \\
\hline & Vicenin-2 (6,8-Di-C-glucosylapigenin) & + & + \\
\hline
\end{tabular}




\begin{tabular}{|c|c|c|c|}
\hline \multicolumn{2}{|r|}{ Phytoconstituents } & \multirow{2}{*}{$\begin{array}{c}\begin{array}{c}\text { Aqueous } \\
\text { artichoke }\end{array} \\
+\end{array}$} & \multirow{2}{*}{\begin{tabular}{|c|}
$\begin{array}{c}\text { Hydro-alcoholic } \\
\text { artichoke }\end{array}$ \\
+ \\
\end{tabular}} \\
\hline \multirow[t]{9}{*}{ Polyphenols } & 1,3-Di-O-caffeoylquinic acid (Cynarin) & & \\
\hline & 5-O-Caffeoylshikimic acid $\mathrm{I}^{\mathrm{a}}$ & + & \\
\hline & 5-O-Caffeoylshikimic acid II & + & \\
\hline & Esculin & + & + \\
\hline & Ferulic acid & + & + \\
\hline & Caffeoylquinic acid Ia & + & \\
\hline & Caffeoylquinic acid IIa & + & \\
\hline & Coumaroylquinic acid la & + & \\
\hline & Coumaroylquinic acid II ${ }^{a}$ & + & \\
\hline \multirow[t]{4}{*}{ Other metabolites } & Indole-4-carbaldehyde & + & + \\
\hline & Choline & + & + \\
\hline & Methyl cinnamate ${ }^{\mathrm{b}}$ & & + \\
\hline & Phenethylamine & + & + \\
\hline \multirow{9}{*}{$\begin{array}{l}\text { Purines and } \\
\text { pyrimidines }\end{array}$} & 5'-S-Methyl-5'-thioadenosine & + & + \\
\hline & Adenine & + & + \\
\hline & Adenosine & + & + \\
\hline & $\begin{array}{l}\text { Adenosine 5'-monophosphate } \\
\text { (AMP) }\end{array}$ & + & \\
\hline & Cytidine & + & + \\
\hline & Guanosine & + & + \\
\hline & $\begin{array}{l}\text { Guanosine 5'-monophosphate } \\
(\text { GMP) }\end{array}$ & + & \\
\hline & Uracil & + & + \\
\hline & Uridine $^{\mathrm{a}}$ & + & \\
\hline \multirow[t]{2}{*}{ Saponins } & Cynarasaponin E & + & + \\
\hline & Cynarasaponin C & + & + \\
\hline \multirow[t]{2}{*}{ Terpenoid } & Cynaratriol & + & + \\
\hline & Ursolic acid $^{\mathrm{b}}$ & & + \\
\hline Sugars & Glucose or Galactose & + & + \\
\hline \multirow[t]{2}{*}{ Steroids } & Stearidonic acid methyl ester ${ }^{\mathrm{b}}$ & & + \\
\hline & Stearidonic acid ethyl ester ${ }^{\mathrm{b}}$ & & + \\
\hline \multirow[t]{5}{*}{ Vitamines } & Nicotinamide & + & + \\
\hline & Nicotinic acid (B3) & + & + \\
\hline & Pantothenic acid (B5) & + & + \\
\hline & Pyridoxal $^{\mathrm{a}}$ & + & \\
\hline & Pyridoxine (B6) & + & + \\
\hline
\end{tabular}

${ }^{\text {a }}$ Compounds to be found only in aqueous extract

${ }^{\mathrm{b}}$ Compounds found only in hydro-alcoholic extracts

According to our current knowledge, we were the first to identify the kynurenic acid, trigonelline and stachydrine as the major alkaloids present in both artichoke extracts. The neuroprotective role of kynurenic acid has been already 
demonstrated, and is achieved via the kynurenine pathway by metabolizing the tryptophan amino acid that is also present in both of ours artichoke extracts [27]. The presence of trigonelline in plant extracts like coffee and fenugreek was demonstrated, and some experimental data did indicate its Nrf2 inhibitory effect together with the blocking of Nrf2-dependent expression of proteasomal genes, and reduced proteasome activity in some pancreatic carcinoma cell lines [28]. Stachydrine is a prolinebetaine type of alkaloid that was suggested to play an important role in prevention of cardiovascular diseases by inhibiting the deleterious effect of high-glucose on endothelial cells through the modulation of SIRT1 pathway [29].

With the exception of phenylalanine and asparagine, all the other amino acids listed in Table 3. are reported for the first time in the case of artichoke extracts [30].

In this paper we are describing also for the first time the presence of some coumarins in artichoke extracts. The newly identified 7-methoxy-4methylcoumarin was shown by others to behave like the multidrug resistant modulator verapamil that was more cytotoxic against tumor cells than normal cells [31]. Cinnamaldehyde is found in both of our artichoke extracts, and it was shown by others to ameliorate the induced cardiac dysfunction in rats by inhibiting ROS production and autophagy through TLR4-NOX4 pathway and exhibits anti-inflammatory activity [32]. Similarly to others [33], we were able to identify the scopoletin in artichoke leaves hydro-alcoholic extracts, and it was suggested to have an important anti-inflammatory activity by inhibiting the phosphorylation of NF-kB and p38 MAPK in mice [34].

Flavonoids like apigenin, apigenin-7-O-glucoside, apigenin-7-Oglucuronide, luteolin-7-O-glucuronide, luteolin-7-O-glucoside and apigenin7-rutinoside had been already reported [35-38]. However, flavonoids like diosmetin, salvigenin, naringin dihydrochalcone and vicenin-2 have been for the first time identified, and are mostly present in the hydro-alcoholic artichoke extract (see Table 3.). Diosmetin was shown by others to inhibit the metastasis of hepatocellular carcinoma cells $[39,40]$, while salvigenin antitumor and immunomodulatory effects on tumor bearing mice had been demonstrated [41]. The naringin dihydrochalcone biological effects were not analysed to present days, however its major constituent the naringin was suggested to be the main component of Ganshuang granule that plays an anti-fibrotic role through deactivation of hepatic stellate cells in cirrhotic mouse model [42], and through the attenuation of EGFR/ERK signalling could suppress cancer cell growth [43]. In the case of vicenin-2 has been recently shown that can suppress high-glucose induced vascular inflammatory processes in human umbilical vein endothelial cells and mice, thereby suggesting its effectiveness as a therapeutic agent for vascular inflammatory diseases [44, 45]. 
The polyphenol content of artichoke was extensively analysed, and several papers were published comparing mature and baby plants in raw or cooked forms with the a relevant phytoconstituent like cynarin -1,3-Di-Ocaffeoylquinic acid [46]. Our aqueous artichoke extract contained much more polyphenols than the hydro-alcoholic extract, and several bioactive constituents were identified for the first time in artichoke, including 5-O-caffeoylshikimic acid, esculin and coumaroylquinic acids (see Table 3.). At present, no data are available regarding the biological effects of 5-O-caffeoylshikimic acid. Esculin has been found to feature gastroprotective effect in mice presumably through the inhibition of NF-KB activation [47], and its protective role against the genotoxicity induced by mitomycin $C$ on liver and kidney mice cells was also described [48]. Ferulic acid is considered the methylated derivate of caffeic acid, and it was suggested that together with other flavonoids and polyphenols to contribute to the antioxidant, anti-inflammatory and anti-septic potential of Lolium multiflorum extracts [49].

Among metabolites we could identify indole-4-carbaldehyde that has not been descried in previously by others, while the incidence of choline, methyl cinnamate and phenethylamine are shown for the first time in the case of artichoke extracts. Methionine- and choline-deficient diet leads to nonhydroalcoholic fatty liver diseases in mouse, rat and swine model systems, therefore, it is expected that the administration of choline would contribute to the prevention of nonhydro-alcoholic steatohepatitis and fibrosis. Methyl cinnamate is a safe antibacterial and flavouring agent used in food industry, and was shown to inhibit the gastrointestinal contractility [50], PPARY activity and adipocyte differentiation in part, by the CaMKK2-AMPK signalling pathway [51]. Phenethylamine is widely used in weight-loss type of dietary supplements [52].

We were able to confirm the finding of others with respect to the presence of saponins like cynarasaponin $\mathrm{C}, \mathrm{E}, \mathrm{B}$ and $\mathrm{K}$ in artichoke extracts $[26,53]$, while their biological effects remained totally elusive.

Among terpenoids the cynaratriol was already reported in artichoke extracts, while the ursolic acid is a newly identified phytoconstituent. The cynaratriol biological effects are not elucidated, while for ursolic acid has been demonstrated to exert anti-oxidative and anti-inflammatory effects on mouse brain injury model by activating the Nrf2-ARE pathway [54], moreover its anti-cancer and anti-metastatic effects were also proven $[55,56]$.

We were also able to identify carbohydrates in artichoke extracts, though the applied method did not allow us to distinguish between glucose and galactose.

According to our current knowledge, steroids like stearidonic acid methyl ester and stearidonic acid ethyl ester were not reported in the case of previously studied artichoke extracts. However, the steroids detected by us 
are derivates of the stearidonic acid (18:4n-3), a plant-derived dietary n-3 PUFA, whose impact on tissue n-3 PUFA content are lacking.

The identification of vitamin $C$ and some vitamins belonging to the $B$ group (thiamine, riboflavine, nicotinamide and nicotinic acid) in artichoke extracts was already reported [57]. It has been demonstrated that the nicotinic acid can inhibit lipolysis, acutely reducing plasma free fatty acid concentrations, and my act in much the same manner as cynarin [58]. We are describing for the first time the incidence of pantothenic acid, pyridoxal and pyridoxine in artichoke extracts, while the above mentioned B5 and B6 vitamins were suggested to act as cancer risk reduction agents [59], and having anti-inflammatory effects associated with atherosclerosis and autoimmunity [60].

During our study, we also came across other phytoconstituents like vitamin $\mathrm{C}$, thiamine, rutin, luteolin and quercetin. The molecular peaks have been identified for the above mentioned phytoconstituents, and the corresponding specific isotopic patterns confirm their molecular structure, but their fragmentation profiles do not corroborate with the values previously reported in scientific literature.

\section{CONCLUSIONS}

In the current paper, we are describing the comparative chemomapping of aqueous and hydro-alcoholic extracts of artichoke leaves. Some previously reported phytoconstituents presence was confirmed, while many other newly identified compounds are reported for the first time to be specific to artichoke. The currently described phytoconstituent profile strongly supports the liver and gallbladder tonic effect of artichoke by interfering with lipid metabolism. Moreover, some kind of anti-cancerous effect could also be expected based on some phytoconstituents. Indeed we were able to demonstrate that the aqueous and hydro-alcoholic extracts of artichoke presented in this paper possess anti-cancerous effects [14]. Based on individual effects of the identified phytoconstituents, multiple mechanisms could be evoked to explain the artichoke health promoting effects like the inhibition of cholesterol synthesis and lipolysis, together with the activation of anti-inflammatory, anti-tumour growth cellular pathways. It seems therefore likely that due to the plethora of phytoconstituents found in artichoke, the health promoting effect of the analysed extracts, might have a more stochastic than determinative nature. Further experiments are needed based on a system biology type of approach to clarify the complexity of the beneficial effects including the correlations with chemical composition. 


\section{EXPERIMENTAL SECTION}

\section{x. Materials and methods}

\section{x.1. Chemicals and reagents}

HPLC-MS grade acetonitrile, water and formic acid were purchased from Fisher Scientific (Geel, Belgium). HPLC grade ammonium acetate and ammonium formate were purchased from Sigma-Aldrich (Munich, Germany).

\section{x.2. Plant material} Hungary.

The artichoke dried leaves were obtained from TTDR 2000 Ltd.,

\section{x.3. Sample preparation}

Aqueous (AE) extract: Artichoke dried leaves $(5 \mathrm{~g})$ were cooked (5 min) in boiling water $(100 \mathrm{ml})$. After cooling at room temperature, the extract was centrifuged (10 min, $4000 \mathrm{rpm}$ ) and filtered through Whatman filter paper (Sigma Aldrich).

Hydro-alcoholic (HE) (ethanol : water 1:1) extract: $50 \mathrm{~g}$ artichoke dried leaves were extracted two times with $500 \mathrm{ml}$ ethanol - water (50:50) by stirring for $4 \mathrm{~h}$ at $40{ }^{\circ} \mathrm{C}$. This artichoke solution were centrifuged at $4000 \mathrm{rpm}$ for $10 \mathrm{~min}$ at room temperature and moved the ethanol from the sample in a rotation vacuum evaporator.

Both types of samples were filtered through a $45 \mu \mathrm{m}$ filter and stored at $4{ }^{\circ} \mathrm{C}$ until analysis.

\section{x.4. HPLC-MS analysis}

The UHPLC system (Dionex Ultimate 3000RS equipped with a Thermo Accucore C18 column, 100/2.1 with a particle size of $2.6 \mu \mathrm{m}$ ) was coupled to a Thermo $Q$ Exactive Orbitrap mass spectrometer equipped with an electrospray ionization source $(\mathrm{ESI})$. Eluent $\mathrm{A}(500 \mathrm{ml}$ of water containing $10 \mathrm{ml}$ of acetonitrile, $0.5 \mathrm{ml}$ of formic acid and $2.5 \mathrm{mM}$ of ammonium formate) and eluent $B(500 \mathrm{ml}$ of acetonitrile containing $10 \mathrm{ml}$ of water, $0.5 \mathrm{ml}$ of formic acid and $2.5 \mathrm{mM}$ of ammonium formate) were used in the HPLC separation in positive ionization mode, and eluent $A$ ( $500 \mathrm{ml}$ of water containing $10 \mathrm{ml}$ of acetonitrile and $2.5 \mathrm{mM}$ of ammonium acetate) and eluent $B(500 \mathrm{ml}$ of acetonitrile containing $10 \mathrm{ml}$ of water and $2.5 \mathrm{mM}$ of ammonium acetate) were used in the HPLC separation in negative ionization mode. Flow rate was $200 \mu \mathrm{l} / \mathrm{min}$. The following gradient elution program was used both positive and negative ionization mode: $0-1 \mathrm{~min}$, 
$95 \%$ A, 1-22 min, $20 \%$ A; $22-24 \min , 20 \%$ A; $24-26$ min, 95\% A; 26-40 min, $95 \%$ A. $5 \mu \mathrm{l}$ of samples were injected in every run. The $Q$ Exactive hybrid quadrupole-orbitrap mass spectrometer was operated with the following parameters: capillary temperature $320{ }^{\circ} \mathrm{C}$, spray voltage $4.0 \mathrm{kV}$ in positive mode and $3.8 \mathrm{kV}$ in negative mode, the resolution was set to 35000 in the case of MS and to 17500 in the case of MS/MS. The mass range scanned was $100-1000 \mathrm{~m} / \mathrm{z}$. Collision energy was $40 \mathrm{NCE}$ in the MS/MS scans. The used UHPLC-ESI-MS measurement accuracy is within 5ppm. The difference between measured and calculated molecular ion masses were always below 5 ppm.

\section{ACKNOWLEDGMENTS}

We would like to thank to the members of the Agricultural and Molecular Research Institute of University of Nyíregyháza for their helpful technical assistance. We are thankful to Dr. Neli Kinga Olah for her comments and suggestions regarding phytoconstituents of artichoke and manuscript preparations. The research expenses were covered by the "In vitro study of some plant extracts of natural origin with emphasis on their anti-tumor effects." HURO/0801 HungarianRomanian Cross Border Cooperation 2007-2013 grant.

\section{REFERENCES}

1. R.E. J.Llorach, F. Tomás-Barberan, F. Ferreres, Journal of Agricultural and Food Chemistry, 2002, 50, 3458.

2. F. Fratianni, M. Tucci, M. De Palma, R. Pepe, F. Nazzaro, Food Chemistry, 2007, 104, 1282.

3. R. Ferracane, N. Pellegrini, A. Visconti, G. Graziani, E. Chiavaro, C. Miglio, V. Fogliano, Journal of Agricultural and Food Chemistry, 2008, 56, 8601.

4. F. Tomás-Barberán, J.C. Espín, Journal of the Science of Food and Agriculture, 2001, 81, 853.

5. M. Lutz, C. Henríquez, M. Escobar, Journal of Food Composition and Analysis, 2011, 24, 49.

6. X. Wu, G. Beecher, J. Holden, D. Haytowitz, S. Gebhardt, R. Prior, Journal of Agricultural and Food Chemistry, 2004, 52, 4026.

7. B.L. Halvorsen, Carlsen M.H., Phillips K. M., Bohn S.K., Jacobs D.R., Blomhoff R. American Journal of Clinical Nutrition, 2006, 84, 95.

8. M. Ben Salem, H. Affes, K. Ksouda, R. Dhouibi, Z. Sahnoun, S. Hammami, K.M. Zeghal, Plant Food for Human Nutrition, 2015, 70(4),441. 
9. M. Rondanelli, F. Monteferrario, S. Perna, M.A. Faliva, A. Opizzi, Monaldi Archives of Chest Disease, 2013, 80 (1), 17.

10. B. Wider, M.H. Pittler, J. Thompson-Coon, E. Ernst, The Cochrane Database of Systematic Reviews, 2013, (3):CD003335.

11. A.M. Mileo, D. Di Venere, C. Abbruzzese, S. Miccadei, Oxidative Medicine and Cellular Longevity, 2015, 363827.

12. C. Pulito, F. Mori, A. Sacconi, L. Casadei, M. Ferraiuolo, M.C. Valerio, R. Santoro, F. Goeman, A. Maidecchi, L. Mattoli, C. Manetti, S. Di Agostino, P. Muti, G. Blandino, S. Strano, Oncotarget, 2015, 6 (20), 18134.

13. E.N. Simsek, T. Uysal, Asian Pacific Journal of Cancer Prevention, 2013, 14 (11), 6791.

14. S. Vígh, C. Pribac, M. Czapár, Z. Zsvér-Vadas, L. Moş, C. Mihali, L. Cziáky, S.T. Sinka, V. Turcuş, E. Máthé, Studia Universitas Vasile Goldiş, 2016, 26 (4), 423.

15. I.M. Abu-Reidah, D. Arráez-Román, A. Segura-Carretero, A. FernándezGutiérrez, Food Chemistry, 2013, 141, 2269.

16. A. Moglia, S. Lanteri, C. Comino, A. Acquadro, R. de Vos, J. Beekwilder, Journal of Agricultural and Food Chemistry, 2008, 56, 8641.

17. M.N. Clifford, W. Zheng, N. Kuhnert, Phytochemical Analysis, 2006, 17, 384.

18. R. Coinu, S. Carta, P.P. Urgeghe, N. Mulinacci, P. Pinelli, F. Franconi, A. Romani, Food Chemistry, 2007, 101, 524.

19. R. Ferracane, N. Pellegrini, A. Visconti, G. Graziani, E. Chiavaro, C. Miglio, V. Fogliano, Journal of Agricultural and Food Chemistry, 2008, 56, 8601.

20. K. Schütz, E. Muks, R. Carle, A. Schieber, Journal of Agricultural and Food Chemistry, 2006, 54, 8812.

21. E. Azzini, R. Bugianesi, F. Romano, D. Di Venere, S. Miccadei, A. Durazzo, The British Journal of Nutrition, 2007, 97, 963.

22. S. Lombardo, G. Pandino, G. Mauromicale, M. Knödler, R. Carle, A. Schieber, Food Chemistry, 2010, 119, 1175.

23. K. Schütz, D. Kammerer, R. Carle, A. Schieber, Journal of Agricultural and Food Chemistry, 2004, 52, 4090.

24. N. Mulinacci, D. Prucher, M. Peruzzi, A. Romani, P. Pinelli, C. Giaccherini, F. F. Vincieri, Journal of Pharmaceutical and Biomedical Analysis, 2004, 34, 349.

25. G. Pandino, F.L. Courts, S. Lombardo, G. Mauromicale, G. Williamson, Journal of Agricultural and Food Chemistry, 2009, 58, 1026.

26. F. Piozzi, M. Paternostro, S. Passannanti, E. Gacs-Baitz, Phytochemistry, 1986, $25,539$.

27. K. Sas, H. Robotka, J. Toldi, L. Vécsei, Journal of the Neurological Sciences, 2007; 257 (1-2), 221.

28. A. Arlt, S. Sebens, S. Krebs, C. Geismann, M. Grossmann, M.L. Kruse, S. Schreiber, H. Schäfer, Oncogene, 2013, 32 (40), 4825.

29. L. Servillo, N. D'Onofrio, L. Longobardi, I. Sirangelo, A. Giovane, D. Cautela, D. Castaldo, A. Giordano, M.L. Balestrieri, Journal of Cellular Biochemistry, 2013, $114(11), 2522$.

30. R. Bernhard, Lebensmittel-Wissenschaft \& Technologie, 1972, 5, 185. 
31. M. Kawase, H. Sakagami, N. Motohashi, H. Hauer, S.S. Chatterjee, G. Spengler, A.V. Vigyikanne, A. Molnár, J. Molnár, In Vivo, 2005; 19 (4), 705.

32. H. Zhao, M. Zhang, F. Zhou, W. Cao, L. Bi, Y. Xie, Q. Yang, S. Wang, Journal of Molecular and Cellular Cardiology, 2016, 101,11.

33. J. Hinou, C. Harvala, S. Philianos, Annales Pharmaceutiques Francaises, 1989, $47(2), 95$.

34. M.V. Pereira Dos Santos Nascimento, F. Arruda-Silva, A.B. Gobbo Luz, B. Baratto, D. Venzke, B.G. Mendes, T.S. Fröde, M. Geraldo Pizzolatti, E.M. Dalmarco, Immunopharmacology and Immunotoxicology, 2016, 38 (5), 344.

35. C. Pereira, L. Barros, A.M. Carvalho, C. Santos-Buelga, I.C. Ferreira, Food and Function, 2015, 6 (1), 56.

36. D. Negro, V. Montesano, S. Grieco, P. Crupi, G. Sarli, A. De Lisi, G. Sonnante, Journal of Food Sciience, 2012, 77(2), C244.

37. J. Magielse, A. Verlaet, A. Breynaert, B. M. Keenoy, S. Apers, L. Pieters, N. Hermans, Molecular Nutrition and Food Research, 2014, 58 (1), 211.

38. M. Wang, J.E. Simon, I.F. Aviles, K. He, Q.Y. Zheng, Y. Tadmor, Journal of Agricultural and Food Chemistry, 2003, 51 (3), 601.

39. K. Patel, M. Gadewar, V. Tahilyani, D.K. Patel, Chinese Journal of Integrative Medicine, 2013, 19, 792.

40. J. Liu, X. Wen, B. Liu, Q. Zhang, J. Zhang, H. Miao, R. Zhu, Molecular Medicine Reports, 2016, 13 (3), 2401.

41. S. Noori, Z. M. Hassan, B. Yaghmaei, M. Dolatkhah, Cellular Immunology, 2013, $286(1-2), 16$.

42. H. Shi, H. Shi, F. Ren, D. Chen, Y. Chen, Z. Duan, Journal of Cellular and Molecular Medicine, 2016, doi: 10.1111/jcmm.12994.

43. A. Yoshinaga, N. Kajiya, K. Oishi, Y. Kamada, A. Ikeda, P. K. Chigwechokha, T. Kibe, M. Kishida, S. Kishida, M. Komatsu, K. Shiozaki, European Journal of Pharmacoogy,. 2016, 782, 21.

44. S.K. Ku, J.S. Bae, Canadian Journal of Physiology and Pharmacology, 2016, 94 (3), 287.

45. H. Kang, S.K. Ku, B. Jung, J.S. Bae, Inflammation Research, 2015, 64(12), 1005.

46. M. Lutz, C. Henzíquez, M. Escobar, Journal of Food Composition and Analysis, 2011, 24, 49.

47. W. Li, Y. Wang, X. Wang, H. Zhang, Z. He, W. Zhi, F. Liu, X. Niu, Fundamental and Clinical Pharmacology, 2016, doi: 10.1111/fcp.12255.

48. I. Mokdad Bzeouich, N. Mustapha, M. Maatouk, K. Ghedira, M. Ghoul, L. ChekirGhedira, Regulatory Toxicology and Pharmacology, 2016, 82, 48.

49. K.C. Choi, Y.O. Son, J.M. Hwang, B.T. Kim, M. Chae, J.C. Lee, Pharmaceutical Biology, 2016, 55 (1), 611.

50. F.J. Lima, F. Cosker, T.S. Brito, H.V. Ribeiro-Filho, C.M. Silva, K.S. Aragão, S. Lahlou, M.H. Souza, A.A. Santos, P.J. Magalhães, European Journal of Pharmacology, 2014, 740, 192.

51. Y.Y. Chen, M.H. Lee, C.C. Hsu, C.L. Wei, Y.C. Tsai, Journal of Agricultural and Food Chemistry, 2012, 60 (4), 955. 
52. R.S. Pawar, E. Grundel, Drug Testing and Analysis, 2016, doi: 10.1002/dta. 1980.

53. P. Mucaji, D. Grancai, M. Nagy, M. Buděsínský, K. Ubik, Ceska a Slovenska Farmacie, 2001, 50 (6), 277.

54. H. Ding, H. Wang, L. Zhu, W. Wei, Neurochemical Research, 2016, doi:10.1007/s11064-016-2077-8.

55. W.T. Gai, D.P. Yu, X. S. Wang, P.T. Wang, Oncology Letters, 2016, 12 (4), 2880.

56. J.L. Gao, Y.M. Shui, W. Jiang, E.Y. Huang, Q.Y. Shou, X. Ji, B.C. He, G.Y. Lv, T.C. He, Oncotarget. 2016. 71802.

57. E. Serni, V. Audino, S. Del Carlo, C. Manera, G. Saccomanni, M. Macchia, Natural Product Research, 2013, 27 (23), 2212.

58. T. Kroon, T. Baccega, A. Olsén, J. Gabrielsson, N.D. Oakes, Journal of Lipid Research, 2017, 58 (1), 31.

59. S. Mocellin, M. Briarava, P. Pilati, Journal of the National Cancer Institute, 2016, 109 (3), pii: djw230.

60. S.C. Gominak, Medical Hypotheses, 2016, 94, 103. 
